\title{
Prolong the Life time of Network using Minimum Energy Consumption in MANET
}

\author{
Hitesh Kumar Rinhayat \\ Department of Computer \\ Science \& Engineering \\ Technocrats Institute of \\ Technology \\ Bhopal (M.P.), India
}

\author{
Aakanksha Jain \\ Department of Computer \\ Science \& Engineering \\ Technocrats Institute of \\ Technology \\ Bhopal (M.P.), India
}

\author{
Kamlesh Chandravanshi \\ Department of Computer \\ Science \& Engineering \\ Technocrats Institute of \\ Technology \\ Bhopal (M.P.), India
}

\begin{abstract}
Mobile Ad hoc network (MANET) are self-configuring, individual nodes or routers networks that move about freely, organize themselves randomly and are interconnected through wireless links that when synchronized, form a dynamic topology. The nodes in required battery power for communication and it is very crucial matter to utilize the battery power of nodes efficiently in network. The battery replacement and charging is not possible in that kind of network and the insufficient battery lifetime imposes a limitation on the network performance. To take full utilization of the lifetime of nodes, traffic should be routed in a way that energy consumption is minimized. The energy efficient routing is improves the battery life and routing capability of nodes. In this research we proposed a new energy efficient routing scheme with AODV routing protocol to improve the reliability of data delivery and energy utilization. The proposed scheme is efficiently utilizes the energy of mobile nodes through variation in the energy of nodes on the basis of their radio range in network utilization. It means the nodes are only communicate to nearest neighbor by that the less amount of transmission and receiving energy is required for communication. In this approach the proposer energy utilization and consumption is possible that reduces the improper energy depletion of mobile nodes. The routing performance is measured through performance matrices and the proposed scheme is showing the much better performance as compare to normal energy routing with AODV routing protocol.
\end{abstract}

\section{Keywords}

Energy, MANET, AODV, routing, performance

\section{INTRODUCTION}

The Mobile Ad hoc Network (MANET) is an independent network established connection and deliver data in network without any supervision of any authority. Mobile ad hoc networks have various civilian and military applications which have drawn considerable attentions in modern area. The major concerns in designing Mobile Ad hoc Networks is to reduce the energy consumption as the wireless nodes are often powered by batteries only. For a sender to send data packets to a destination that is not in its direct radio range of transmission, the packets must be spread through one or more intermediate nodes [1,2]. Ad hoc network nodes in most portable devices, limited by the size of the battery-powered, the whole network is an energy-bounded systems, to save the energy of nodes as much as possible and to maximize the total battery life of a wireless network [3] we must minimize the energy consumption of the entire network using intermediate node minimum energy consumption base. This is particularly important in emergency rescue, military operations, business meetings and other situations. From this perspective, the shortest route is not necessarily the best route. On the contrary, with some short hop configurations to replace the relatively long jump configurations may be better energy-saving choices available [4].

At present, there are two main ideas of energy-saving routing algorithms for Ad hoc routing protocols [5]. The first one is to send each packet with minimum energy-consuming. The second is to maximize the network lifetime as much as possible. These two ideas are considered relatively independent of one another, the intention is to combine these two ideas, and to therefore maximize the network's lifetime and minimize energy consumption.

\section{RELATED WORK}

In this section we study about existing energy aware routing and its benefits, that related work encourage to work the newer research in the field of energy issue in MANET and solve the problem occur on existing system.

Sheetal Sisodia et al. [1] in this work comparing two different protocols in context of energy conservation and routing overhead. The proposed work is done in two modules named Node Energy Aware Methodology which includes conditional Min-Max Battery Cost Routing Algorithm (CMM-BCR) and Destination Estimation Module which includes Distance Routing Effect Algorithm for Mobility (DREAM) location based protocol. These two modules together applied over OnDemand Routing protocol which is a Dynamic Source Routing protocol (DSR) and over Destination Sequence Distance Vector Routing Protocol (DSDV) which is a Table driven routing protocol. Simulation shows that when this energy scheme used with DSR provides better result than DSDV. As energy is of main concern in ad-hoc networks so our main aim is to know which among these two protocols is good in energy conservation and increases network lifetime by reducing overhead.

Mr. Varun Mishra et. al. has proposed [6] a "Energy Aware and Multipath base Reliable Communication in MANET" in this mechanism they propose a multi-path MAX energy based routing scheme that reduces the congestion and improves the energy efficiency and the reliability in data delivery. In this mechanism the multipath routing protocol reduces the possibility of congestion by using the concept of dynamic queue and MAX energy based routing always selecting the node for routing that has maximum energy. Every data packet is delivered to the neighbour by one or more multiple paths according to their proposed scheme. The stability among multiple paths that considers the energy usage at neighbours is further considered in path selection, which leads to competent utilization of the spread nodes and prevents early death of 
heavily involved nodes, in that mechanism they solve congestion problem with the help of AOMDV routing and reliable data delivery using maximum node energy base routing but they can't solve minimum energy utilization of energy of network so further we improve that mechanism by our newer energy aware with minimum transmission as well as receiving power requirement base technique and provide the strength of that existing approach.

AL-Gabri Malek et. al [7] in his work titled "New Energy Model: Prolonging the Lifetime of Ad-hoc On-Demand Distance Vector Routing Protocols (AODV)" In this approach they discusses a new energy model that prolongs the lifetime of Ad-hoc On-Demand Distance Vector Routing Protocols which are modified to improve the networks lifetime in MANETs. In this mechanism they are proposed two main ideas of energy saving routing algorithm for Ad hoc routing protocols: The first one is to send each data packet with minimum energy utilization. The second is to maximize the network lifetime as much as possible. According to energy, the shortest path selection may not be the best path. In this new mechanism of energy-aware named EA_AODV is proposed, which is based on the classical AODV. In that proposal we solve the problem of minimum energy consumption in further we also enhanced that mechanism by the threshold base energy aware scheme and provide sender data duration base data delivery and reliability data grunted scheme.

M.Tamilarasi et. al [8] has been proposed "Efficient Energy Management for Mobile Ad Hoc Networks" in this mechanism they proposed an algorithm for modifying DSR to reduce overhead by reducing the number of route reply packets and the header size of DSR data packets. Besides this an algorithm for energy management is incorporated in the Modified DSR by transmitting the data packets with minimum required energy. That proposal useful for minimum energy requirement under DSR routing, but DSR routing work minimum hop base mechanism not time delay base so further we improve that proposal by the help of shorter delay techniques.

Nivedita N. Joshi et. Al [9] in his work titled "Energy Conservation in MANET Using Variable Range Location Aided Routing Protocol" proposed a scheme to controls the transmission power of a node according to the distance between the mobile nodes. It also includes energy information on route request packet and selects the energy efficient path to route data packets. and study the behaviour of energy as well as location aware routing base approach that is efficient with power reduction mechanism but overhead is maximum so in future we improve that work on the base of minimum overhead base technique.

Rana Alhalimi et. al has been proposed [10] "Energy Level Accuracy in Mobile Ad-Hoc Networks Using OLSR" In this title they use Optimized Link State Routing (OLSR) as the underlying routing protocol. They report the quantification of state information accuracy under different traffic rates. They are focusing on energy level as Quality of Service metric, which has been used for routing decisions in many energyefficient routing protocols. State information accuracy is defined as the average difference between perceived energy level and its actual value. That work useful for proactive routing where node motion is very limited so further we improve that work with the help of reactive routing protocol.

Wei Liu et. al has proposed "DELAR: A Device-Energy-Load Aware Relaying Framework for Heterogeneous Mobile Ad Hoc Networks" [11] and researcher focus work on the crosslayer designed Device-Energy-Load Aware Relaying framework, named DELAR, to accomplish energy conservation from multiple facets, including energy-aware routing, transmission scheduling and energy control. In particular, they design a novel power-aware routing protocol that satisfactorily incorporates device heterogeneity, nodal remaining energy information and nodal load status to save energy. In addition, they develop a hybrid transmission scheduling mechanism, which is a mixture of reservation-based and contention-based medium access control schemes, to coordinate the data transmissions. Moreover, the novel notion of "mini-routing" is introduced into the data link layer and an Asymmetric MAC (A-MAC) scheme is proposed to support the MAC-layer acknowledgements over unidirectional links caused by asymmetric transmission power levels between powerful nodes and normal nodes. Furthermore, they present a multi-packet transmission scheme to improve the end-to-end delay performance.

Ying Zhu et. al has been proposed a "Energy-Efficient Topology Control in Cooperative Ad Hoc Networks" [12] in this work researcher introduce a new topology control problem: name is energy-efficient topology control problem with cooperative communication, and proposed two topology control algorithms to build cooperative energy spanners in which the energy efficiency of individual paths are guaranteed. Both suggested algorithms can be performed in distributed and localized fashion while maintaining the globally efficient paths by proposed mechanism and control the topology change behaviour on the bases of energy efficient mechanism.

Vinay Rishiwal et. al [13] in his work titled "Power Aware Routing in Ad Hoc Wireless Networks" they propose an efficient algorithm and maximizes the network lifetime by minimizing the power consumption during the source to destination route establishment. As on their case study proposed algorithm has been incorporated along with the route discovery procedure of AODV and by simulation it is observed that proposed algorithm's performance is enhanced as compare to AODV and DSR in terms of various energy base parameters like total Energy Consumption, Average Energy Left Per Alive Node, Node Termination Rate, and Network Lifetime for different network scenarios.

\section{PROPOSED SCHEME FOR ENHANCE ENERGY UTILIZATION}

Finally, in this approach we apply minimum power requirement base connection establishment mechanism that provides efficient way communication between senders to receiver. Here initially get energy of each node, transmission as well as receiving power requirement of each node and routing protocol, after that if sender wants to communicate with receiver node than sender initiate route call message and broadcast them that packet received by any node, very first check is a receiver or a forwarder, if that node as a forwarder than calculate energy displacement by given formula and forward the packet to next hop, at the end if we get receiver node by more than one path with loop freedom, than compare both path power requirement of single packet and select minimum power requirement path for further utilization.

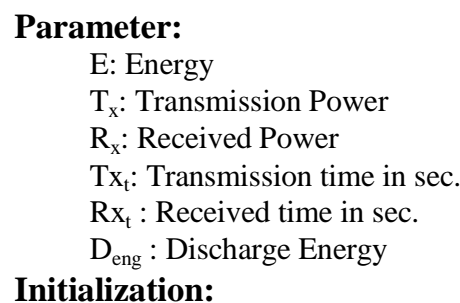




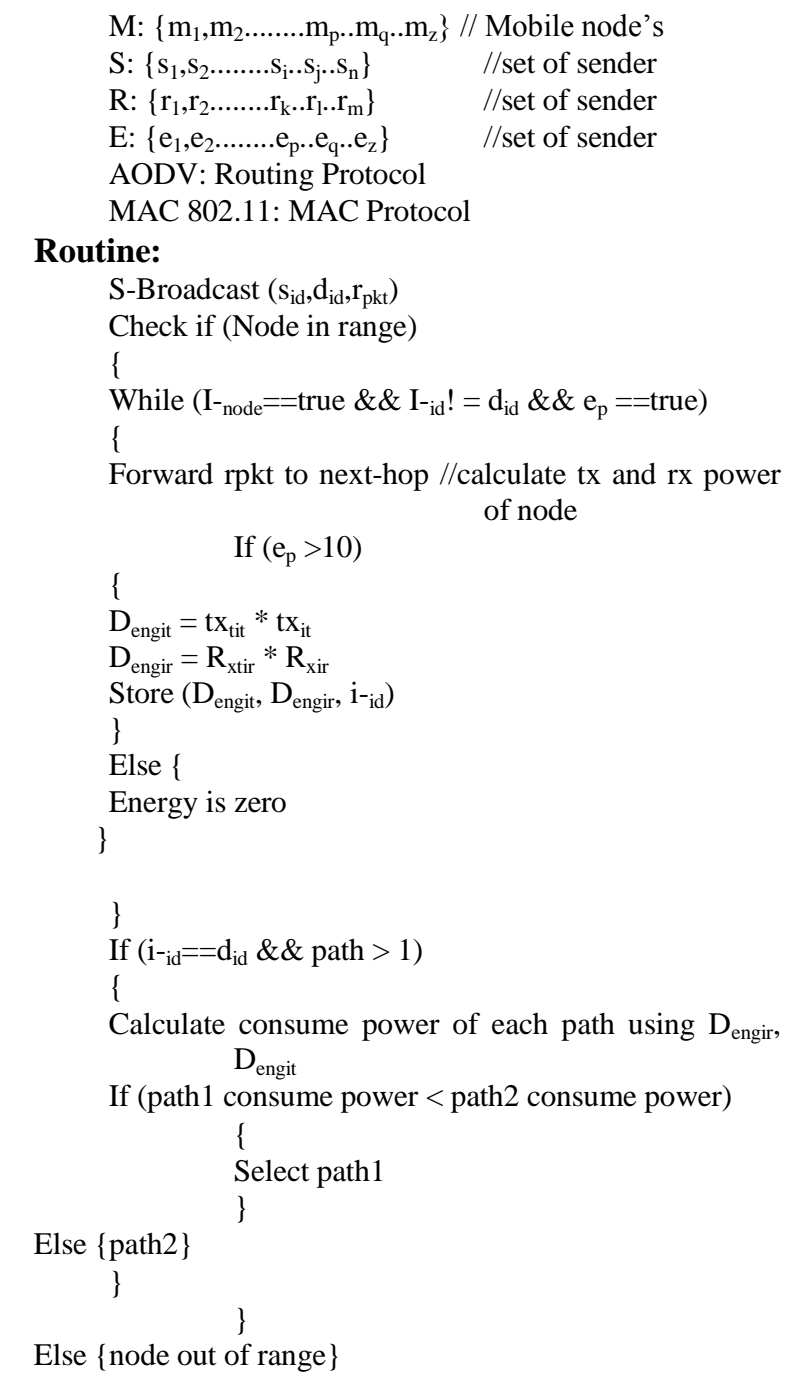

\subsection{Algorithm for Route Repair}

Parameter:

$\mathrm{D}_{\text {engit }}$ : Discharge Energy in case of transmission power

$\mathrm{D}_{\text {engir }}$ : Discharge Energy in case of receiving power

Routine:

If (established path break)

\{

Find reason of disable

If ( $\mathrm{i}_{\text {- }}$ nde of established path $\mathrm{e}_{\mathrm{p}}==0$ )

\{

module

Search new

\} route from $\mathrm{S}$ to $\mathrm{R}$ node

Call routine of initial

Else \{node out of range

\}

Stop

\section{SIMULATION ENVIRONMENT \& RESULTS ANALYSIS}

This section represents the overview of simulator tool and discuss about the results that evaluated after applying the proposed scheme.

\subsection{Overview of Network Simulator}

NS2 is a free simulation tool, which can be obtained from [15]. It runs on various platforms including UNIX (or Linux), Windows, and Mac systems. Being developed in the UNIX environment, with no surprise, NS2 has the fluent ride there, and so does its installation. Unless otherwise specified, the addressed in this book is based on a Cygwin (UNIX emulator) activated Windows system.

NS2 source codes are distributed in two forms: the all-in-one suite and the component-wise. With the all-in-one kit, users get all the required components along with some elective components. This is basically a recommended choice for the beginners. This package provides an "install" script which configures the NS2 environment and creates NS2 executable file using the "make" utility.

\subsubsection{Simulation Parameters}

The following simulator parameters are used to evaluate the performance of normal energy with AODV and proposed energy efficient scheme with AODV is mentioned in table 1.

Table 1.Simulation Parameters for Case Study

\begin{tabular}{|l|l|}
\hline Number of nodes & 50 \\
\hline Dimension of simulated area & $800 \times 600$ \\
\hline Routing Protocol & AODV \\
\hline Simulation time (seconds) & 50 \\
\hline Transmission Range & $250 \mathrm{~m}$ \\
\hline Transport Layer Protocol & TCP, UDP \\
\hline Traffic type & FTP, CBR \\
\hline Packet size (bytes) & 512 \\
\hline Number of traffic connections & 12 \\
\hline Maximum Speed $(\mathrm{m} / \mathrm{s})$ & 30 \\
\hline
\end{tabular}

\subsection{Result analysis}

\subsubsection{Packet Delivery Ratio (PDR) Analysis}

The packet delivery ratio (PDR) analysis is measured through the packets percentage receiving at destination in a given simulation time in network. The percentage analysis is completely depending on the ratio of numerator and denominator. This graph exemplified the PDR analysis in case of normal energy routing and proposed energy efficient routing in network that minimizes the energy consumption in network. The PDR in normal routing is about $87 \%$ but in case of proposed scheme is about $92 \%$ in network at the end of simulation. The difference of $5 \%$ represents that the improvement in percentage of data receiving. The proposed scheme utilizes the maximum amount of energy in data delivery in network by that the packets receiving is improves in network i.e. also enhance the network performance.

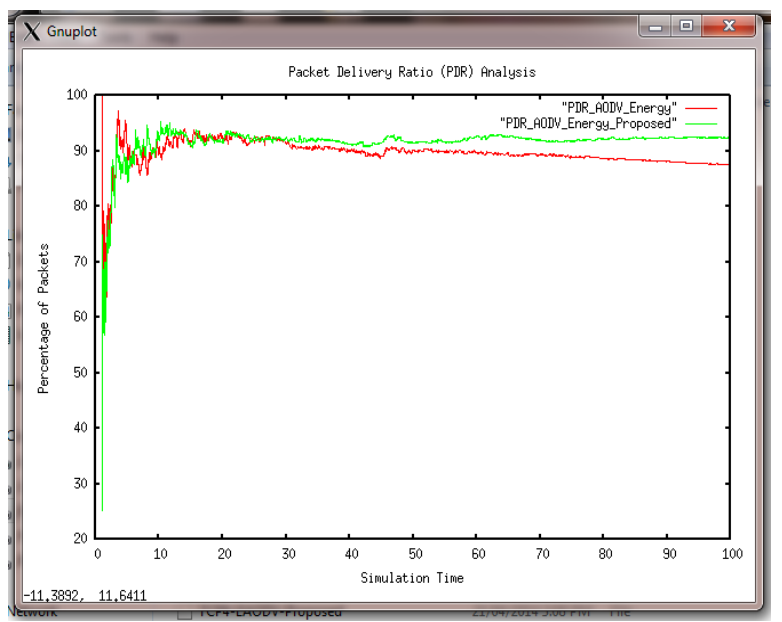

Fig. 2 PDR Analysis 


\subsubsection{Routing Load Analysis}

The routing load is measured in network on the basis of ratio of number of control packets and the data packets received in network in a given simulation time. The more number of data packets are showing the better performance in network but the packets receiving is down w.r.t. to control packets delivery then in that case more amount of control packets are required to deliver data to receiver. This graph exemplified the routing load or control overhead analysis of normal energy routing and proposed energy efficient scheme with AODV routing protocol. The proposed scheme is utilizes the energy consumption in network by that the less amount of control packets i.e. about 4800 are deliver in network but in case of normal energy routing more than 10,000 packets are deliver in network. That means the more amount of energy is consumed in network for retransmission by that the routing overhead increases and limited battery power utilization decreases in network.

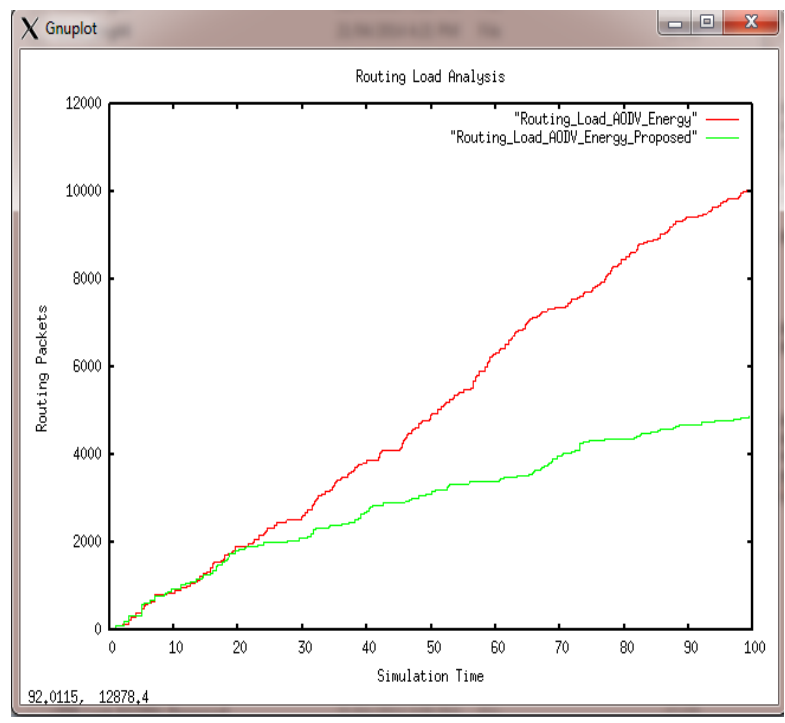

Fig. 2 Routing Load Analysis

\subsubsection{Packets Received per unit of Time Analysis}

The packets receiving in network per unit of time is also called throughput in network. The throughput is evaluated to identify the packets delivery with respect to given simulation time in network. In this graph we analyze the throughput in case of normal energy routing and proposed energy efficient routing. The throughput in case of proposed scheme is about 850 packets/second received in network by receivers but in case of normal energy routing the throughput is about 400 packets / second at the end of simulation time in network. The proposed scheme control the transmission and receiving energy of nodes that removes the possibility of early depletion of mobile nodes in network by that the nodes are reliable for communication and improves the energy depletion and enhance the network performance.

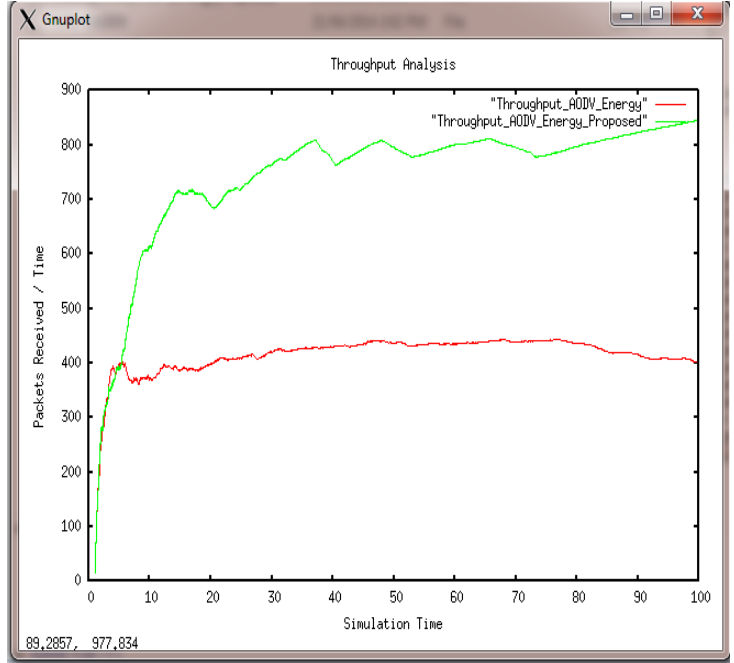

Fig. 3 Throughput Analysis

\subsubsection{Died Node Analysis of Nodes in Normal Routing and Proposed Routing}

The number of no nodes died in network or completely failure for communication in a given time is mentioned in Table 2 . The nodes are utilizes their energy for communication but in proposed energy efficient scheme not a single node is died in network it implies that the nodes having a sufficient amount of energy and not reaches to completely discharge level.

Table 2.Died Node Analysis

\begin{tabular}{|c|c|c|c|}
\hline \multicolumn{2}{|c|}{ Normal Routing } & \multicolumn{2}{c|}{ Proposed Routing } \\
\hline Time & Node No & Time & Node No \\
\hline 57.62497 & 4 & - & - \\
\hline 97.38806 & 5 & - & - \\
\hline 62.14967 & 6 & - & - \\
\hline 80.92668 & 8 & - & - \\
\hline 81.96179 & 22 & - & - \\
\hline 95.0284 & 23 & - & - \\
\hline 54.27771 & 25 & - & - \\
\hline 77.81074 & 26 & - & - \\
\hline 12.0624 & 29 & - & - \\
\hline 29.08781 & 30 & - & - \\
\hline 34.31015 & 31 & - & - \\
\hline 65.36517 & 32 & - & - \\
\hline 48.54182 & 35 & - & - \\
\hline 51.58944 & 36 & - & - \\
\hline 73.85143 & & & - \\
\hline
\end{tabular}

\subsubsection{Overall Summarized Analysis of Energy $A O D V$ and Proposed Energy AODV}

The overall summarized analysis represents the exact network performance i.e. evaluated by performance matrices in case of AODV routing protocol with energy and proposed energy efficient scheme with AODV is mentioned in table 3. The performance of proposed scheme is much better in network because of utilizes the energy for data communication in network but in normal energy with AODV utilizes the more 
amount of energy in retransmission because of link breakage so, the data receiving in network is about $60 \%$ as compare to proposed routing scheme.

Table 3. Summarized Analyses

\begin{tabular}{|l|l|c|}
\hline \multicolumn{1}{|c|}{ Matrices } & $\begin{array}{c}\text { Energy } \\
\text { AODV }\end{array}$ & $\begin{array}{c}\text { Proposed } \\
\text { Energy AODV }\end{array}$ \\
\hline SEND & 3206 & 5657 \\
\hline RECEIVE & 2805 & 5232 \\
\hline ROUTINGPKTS & 10038 & 4879 \\
\hline PDF & 87.49 & 92.49 \\
\hline NRL & 3.58 & 0.93 \\
\hline DROPRTS & 986 & 272 \\
\hline No. of dropped data & 401 & 425 \\
\hline
\end{tabular}

\section{CONCLUSION}

You in Mobile Ad hoc Network, two nodes communicate either directly or indirectly through other nodes and the aim is only one to deliver the data successfully in network. These nodes are typically powered by batteries with limited energy supply. When a node exhausts its available energy then in that condition it stops their functioning. This failure may potentially result in partitioning of the entire network. The limited battery power in MANET is the crucial issue and their utilization is also necessary to improve the routing capability. Different study suggests different techniques to handle energy issue in different way. Energy efficiency continues to be a key performance metric as efficient utilization of energy increases the network longevity hence critical in enhancing the network capacity. So this research effort is made to reduce the energy consumption through proposed scheme. The proposed energy saving scheme aim is to minimizing the total power consumption of all nodes in the group i.e. minimizes the number of nodes used to establish connection, minimize the control overhead and so on and at maximizing the life span should be considered. The simulation results are shows that the proposed scheme is minimizes the energy consumption in retransmission and utilizes the energy for data delivery that enhances the network performance and degrades the possibility of link failure.

\section{REFERENCES}

[1] Sheetal Sisodia, Sandeep Raghwanshi,"Performance Evaluation of a Table Driven and On-Demand Routing Protocol in Energy Constraint MANETs" IEEE International Conference on Computer Communication and Informatics (ICCCI -2013), pp. Jan. 04 - 06, 2013, Coimbatore, INDIA.

[2] J. Lian., L. Li., and X. Zhu., "A Multi-Constraint QoS Routing Protocol with Route- Request Selection Based on
Mobile Predictibg in MANET," IEEE CISW'07, pp. 342 345, Dec. 2007.

[3] Internet Engineering Task Force (IETF).Mobile Ad-hoc Networks (MANET) WORK- GROUP. http://www.ietf.org/html.charters/manet-charter.html.

[4] Stephan Hengstler, Energy-Aware Routing in Wireless Ad Hoc and Sensor Networks, Paper Survey 2 for EE 360 Advanced Topics in Wireless Communications -Spring 2004 - Restore

[5] Cai, W. y. Jin, X. y. Zhang, Y. Chen, K. s. A loadbalanced minimum energy routing algorithm for Wireless Ad Hoc Sensor Networks, 2006, Vol 7; Number 4, pages 502-506.

[6] Mr. Varun Mishra and Mr. Gajendra Sonker, Energy Aware and Multipath base Reliable Communication in MANET, International Journal of Innovative Research in Computer and Communication Engineering, ISSN (Online): 2320 - 9801, Vol. 1, Issue 5, July 2013

[7] AL-Gabri Malek, Chunlin Li, Li Layuan and WangBo, New Energy Model: Prolonging the Lifetime of Ad-hoc On-Demand Distance Vector Routing Protocols (AODV), 978-1-4244-5824-0, IEEE-2010

[8] [8] M.Tamilarasi, S.Chandramathi and T.G. Palanivelu, Efficient Energy Management for Mobile Ad Hoc Networks, Department of Electronics and Communication Engineering Pondicherry Engineering College, Pondicherry, India

[9] Nivedita N. Joshi and Radhika D. Joshi, Energy Conservation In MANET Using Variable Range Location Aided Routing Protocol, International Journal of Wireless \& Mobile Networks (IJWMN) Vol. 3, No. 5, October 2011

[10] Rana Alhalimi and Thomas Kunz, Energy Level Accuracy in Mobile Ad-Hoc Networks Using OLSR, WICON 2007, October 22-24, 2007, Austin, Texas, USA.

[11] Wei Liu, Chi Zhang et. al, DELAR: A Device-EnergyLoad Aware Relaying Framework for Heterogeneous Mobile Ad Hoc Networks, IEEE journal on selected areas in communications, vol. 29, no. 8, september 2011

[12] Ying Zhu, Minsu Huang, Siyuan Chen, and Yu Wang, Energy-Efficient Topology Control in Cooperative Ad Hoc Networks, ieee transactions on parallel and distributed systems, vol. 23, no. 8, august 2012

[13] Vinay Rishiwal , Mano Yadav, S. Verma and S. K. Bajapai, Power Aware Routing in Ad Hoc Wireless Networks, JCS\&T Vol. 9 No. 2, October 2009

[14] The Network Simulator - ns-2. [Online]. Available: http://www.isi.edu/nsnam/ns/. 